\title{
Effect of polymer melt wall slip on the flow balance of profile extrusion dies
}

Cite as: AIP Conference Proceedings 1526, 59 (2013); https://doi.org/10.1063/1.4802603

Published Online: 19 April 2013

Olga S. Carneiro, Luís L. Ferrás, Fernando T. Pinho, and João M. Nóbrega

\section{ARTICLES YOU MAY BE INTERESTED IN}

Stick and slip phenomena during extrusion of polyethylene melts as investigated by laserDoppler velocimetry

Journal of Rheology 44, 413 (2000); https://doi.org/10.1122/1.551092

Temperature induced dimensional variation in extrusion

AIP Conference Proceedings 1779, 030005 (2016); https://doi.org/10.1063/1.4965475

Numerical Analysis of Extrudate Swell in Viscoelastic Materials with Yield Stress

Journal of Rheology 33, 989 (1989); https://doi.org/10.1122/1.550042

\section{Lock-in Amplifiers up to $600 \mathrm{MHz}$

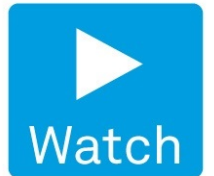

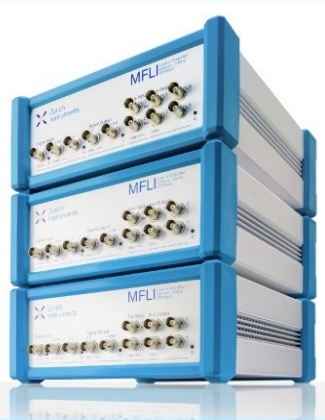




\title{
Effect of Polymer Melt Wall Slip on the Flow Balance of Profile Extrusion Dies
}

\author{
Olga S. Carneiro, Luís L. Ferrás, Fernando T. Pinho, João M. Nóbrega \\ IPC/I3N-Institute Polymers and Composites, Department of Polymer Engineering University of \\ Minho, Campus de Azurém, 4800-058 Guimarães, Portugal
}

\begin{abstract}
This work describes the implementation of the wall slip boundary condition in an in-house developed 3D numerical code based on the Finite Volume Method. For this purpose, several phenomenological models relating the velocity and the shear stress at the wall were implemented. This new feature is verified using a simple case study, by comparing the numerical results with those obtained through the corresponding analytical solution. Then, the potentialities of the new code are illustrated performing flow simulations of a polymer melt in a complex flow channel. The results obtained show that the slip at the wall influences the flow distribution at the die flow channel outlet. Therefore, and to assess the relevance of slippage in the optimal die geometry, the automatic optimization of a die flow channel, required for the production of a specific thermoplastic profile, is performed using both the no-slip and slip boundary conditions, together with two alternative optimization strategies. It is shown that slip favors the flow balance of the dies and also other issues of its performance.
\end{abstract}

Keywords: Wall slip, Flow balance, Extrusion die design.

PACS: 83.50.Rp, 47.11.-j, 81.20.Hy

\section{INTRODUCTION}

In the past, the design of extrusion dies was usually based on experimental trialand-error procedures, which strongly depend on the knowledge and experience of the designer and often require a large number of iterations. During the last decades, the progressive development of computational rheology codes and of computer technology (enabling the implementation of more realistic complex rheological models together with more accurate discretization and interpolation schemes) allowed the progressive replacement of the empirical experimental based design process by computational based systematic methods. This evolution leads to significant savings in time, human and material resources and ensures a better final performance for the tool. The present research team successfully implemented and verified an optimization methodology [1], which encompasses the numerical solution of linear momentum and energy equations by a Finite-Volume Method (FVM) based three-dimensional code, aimed at balancing automatically the flow in profile extrusion dies. In a first stage, the no-slip boundary condition at the flow channel walls was assumed. In fact, the majority of studies concerning the flow in extruders, extrusion dies and rheometers, usually proceed from the assumption that the flowing melt adheres to the wall. However, there are certain plastic melts such as poly(vinyl chloride) (PVC), highdensity polyethylene (HDPE), polypropylene (PP) and elastomers, among other

Novel Trends in Rheology V

AIP Conf. Proc. 1526, 59-71 (2013); doi: 10.1063/1.4802603

(C) 2013 AIP Publishing LLC 978-0-7354-1151-7/\$30.00 
thermoplastics often used in the extrusion of profiles, that show wall-slipping under certain flow conditions. There are well established experimental protocols to adopt in the characterization of this type of behavior when using capillary rheometers without considering the effect of pressure [2] and including this correction (slip) [3] (rotational rheometers [2] and sliding plate rheometers [4]), but, this feature is rarely taken into account in the design of extrusion dies. However, the slip of the fluid at solid walls has a profound influence in the velocity profile, post-extrusion phenomena and on-set of rheological defects and also affects the relationship between integral quantities such as pressure drop and flow rate. According to these facts, the implementation of slip models in computational codes used to simulate the fluid flow is important and useful, both on industrial or scientific contexts, in order to achieve more realistic and accurate results.

As mentioned above, in the previous codes developed by this research team $[1,5]$, the no-slip boundary condition was considered. In the present contribution, this procedure is extended to deal with slip at the solid walls. At this stage generalized Newtonian fluids are considered, but presently it is also being extended to viscoelastic models $[6,7,8]$.

In computational rheology, the Navier-Stokes system of equations (Eq. 1-2) is a unique tool to predict the behavior of complex flows:

$$
\begin{gathered}
\nabla \cdot \mathbf{u}=0 \\
\rho \frac{\partial \mathbf{u}}{\partial t}+\rho \nabla \cdot \mathbf{u u}=-\nabla p+\nabla \cdot \boldsymbol{\tau}+\rho g \\
\mathbf{u}=0
\end{gathered}
$$

Eqs. 1 and 2 are the continuity and the momentum equations, respectively, Eq. 3 is the boundary condition that ensures that no fluid is passing through the walls $(\mathbf{u} \cdot \mathbf{n}=0)$, and that the velocity of the fluid near the wall is nil $(\mathbf{u} \cdot \mathbf{t}=0)$; $\mathbf{u}$ is the velocity vector, $\rho$ is the fluid density, $p$ is the pressure, $\boldsymbol{\tau}$ is the deviatoric stress tensor, $g$ is the gravity, and, $\mathbf{n}$ and $\mathbf{t}$ are the normal and tangential unit vectors. This model, like several other important models, is based on differential equations, and so, it is always necessary to specify boundary conditions, in order guarantee the wellposedness of the problem. Although sometimes there is no clue about which boundary condition to choose and/or to impose (e.g. the boundary condition for the fluid flow through a domain outlet), however in order to obtain a solution, due to mathematical based requirements, we are still forced to state that condition. For this particular case (boundary at the outlet) it is common to use the "do nothing" boundary conditions [9], but which condition to use for the interaction between the liquid and solid walls? If we do a survey through the remarkable books of the last century in the field of fluid dynamics, we can find that almost all of them state that the boundary condition to be used (for the fluid in contact with solid walls) is the Dirichlet boundary condition (Eq. 3), which imposes that the fluid adheres to the wall, and that there is no fluid passing through the walls. One thing that is usually forgotten is that this is an 
assumption that cannot be derived from first principles [10-12]. In some few books of fluid dynamics the authors discuss this topic, whether to say that a slip boundary condition seems wrong or that there is a possibility for the fluid to slip [13-15]. The main reason for the dominance of the no-slip boundary condition was the agreement between experimental works (which were sometimes done with not so accurate measuring instruments, leading to dubious results) and the developed theories. Nowadays, there are many works related to the existence and measurement of the slip velocity in polymer flows as, for example, [2-4,16-19], among others, which reinforce the existence of slip in the liquid-solid interface and result in a comprehensive description of the phenomenon and in the consequent development of phenomenological models to handle it.

In order to include the wall slip boundary condition, Eq. 3 must be replaced by a slip velocity model, such as the well known Navier slip boundary condition [20-22]:

$$
\mathbf{t} \cdot \mathbf{u}=-k \mathbf{t} \cdot \boldsymbol{\tau} \cdot \mathbf{n}
$$

Several other slip models can be found in the literature, and we have chosen the nonlinear Navier slip model,

$$
\|\mathbf{t} \cdot \mathbf{u}\|=-k_{n l}\|\mathbf{t} \cdot \boldsymbol{\tau} \cdot \mathbf{n}\|^{m}
$$

where the slip velocity varies nonlinearly with the tangent stress ( $m$ is the slip exponent and \|\| stands for the usual $l^{2}$ norm) and the asymptotic slip model that is also used by the ANSYS POLYFLOW CFD commercial code [23],

$$
\|\mathbf{t} \cdot \mathbf{u}\|=k_{a 1} \ln \left(1+k_{a 2}\|\mathbf{t} \cdot \boldsymbol{\tau} \cdot \mathbf{n}\|\right)
$$

which assumes that the slip velocity has a logarithmic dependence on the wall tangent stress.

In this work, the implementation of the slip boundary condition at the flow channel walls in an in-house developed 3D finite volume numerical code is described, and, assessed in Section 2 (implementation and assessment). Also the potentialities of the new numerical code are illustrated in this section, by performing flow simulations in complex flow channels. In Section 3 the optimization methodology developed to automatically balance the flow in profile extrusion dies is briefly described and then used in Section 4 (case study) to assess the effect of slippage in the optimal die geometry required for the production of a specific thermoplastic profile. The results obtained are then presented and discussed in Section 5, being the corresponding conclusions drawn in Section 6. 


\section{IMPLEMENTATION AND ASSESSMENT}

The 3D flow fields are calculated with a computational code based on the finite volume method previously developed [5]. It comprises a set of routines to model the relevant physical process and uses the collocated grid arrangement, in which all dependent variables are located at the centre of the control volumes. This greatly simplifies the adoption of general curvilinear coordinates for the mesh [23], being the Cartesian coordinates $\left(x_{1}, x_{2}, x_{3}, t\right)$ converted into general curvilinear coordinates $\left(\xi_{1}, \xi_{2}, \xi_{3}, l\right)$ that fit to the complex geometry using the transformations [24]: $x_{1}=x_{1}\left(\xi_{1}\right.$, $\left.\xi_{2}, \xi_{3}, \imath\right), x_{2}=x_{2}\left(\xi_{1}, \xi_{2}, \xi_{3}, l\right), x_{3}=x_{3}\left(\xi_{1}, \xi_{2}, \xi_{3}, l\right), t=t(\imath)$.

The main modifications performed in the numerical code in order to account for slippage are related to the stress term in the momentum equation (Eq. 2) for the generalized Newtonian fluid. The components of the stress tensor can be written in a generic way as:

$$
\tau_{i j}=\frac{\mu}{J}\left(\beta_{l j} \frac{\partial u_{i}}{\partial \xi_{l}}+\beta_{l i} \frac{\partial u_{j}}{\partial \xi_{l}}\right)-\frac{2}{3} \frac{\mu}{J} \delta_{i j} \beta_{l k} \frac{\partial u_{k}}{\partial \xi_{l}}
$$

where $i, j, k=1,2,3$ are the Cartesian directions, $\beta_{l j}$ is the cofactor of the $l j$ position in the Jacobian (of the coordinate transformation) matrix and $l=1,2,3$ are the directions on the new coordinates. The discretization of the above equation results in:

$$
\tau_{i j}^{f}=\left(\frac{\mu}{\delta V}\right)^{f}\left(\sum_{l=1}^{3}\left(b_{l j}^{f}\left[\Delta u_{i}\right]_{l}^{f}+b_{l i}^{f}\left[\Delta u_{j}\right]_{l}^{f}\right)-\frac{2}{3} \delta_{i j} \sum_{l=1}^{3} \sum_{k=1}^{3} b_{l k}^{f}\left[\Delta u_{k}\right]_{l}^{f}\right)
$$

where $f=1,2,3,4,5,6$ is related to each face of the hexahedral elementary cell. It should be noted that after the discretization, the derivatives, e.g. $\partial u_{i} / \partial \xi_{l}$, are transformed in a finite difference, e.g. $\left[\Delta u_{i}\right]_{l}^{f}$.

The problem is solved in an iterative way, being necessary to define the initial values for the variables. In this case, the velocity field near the flow channel wall must be set. When the no-slip boundary condition is used, a nil tangential velocity is employed, but the velocity at the wall does not need to be nil. In fact, any constant real value can be set for the initial velocity field since while evaluating velocity differences along the face, a nil variation is achieved (if $u_{i}=c n t$ then $\left[\Delta u_{i}\right]_{l}^{f}=0$ ). On the other hand, when the slip boundary condition is used, the iterative scheme illustrated in Figure 1 is adopted. The process will stop when convergence is achieved. 


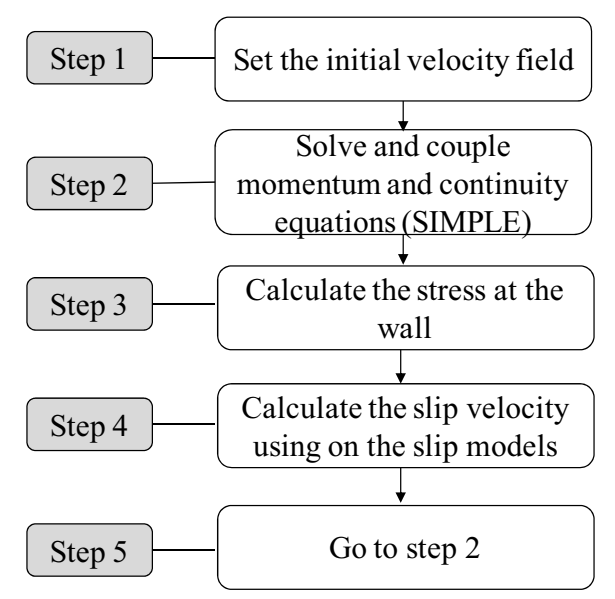

FIGURE 1. Iterative scheme used to compute the slip velocity.

For the assessment of this code we assume a simple two dimensional, stationary, unidirectional (along the $x$ direction), fully developed flow (Figure 2) governed, therefore, by the following equation:

$$
\frac{\partial}{\partial x}\left(\mu \frac{\partial u}{\partial y}\right)=\frac{\partial P}{\partial y}=c t e
$$

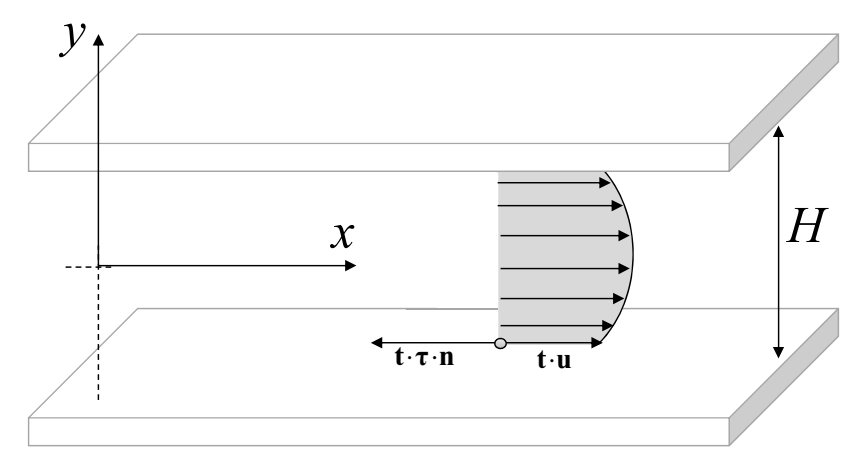

FIGURE 2. Flow between parallel plates.

The solution for Eq. 9 can simply be obtained by imposing an average velocity $U_{1}$,

$$
U_{1}=\frac{1}{H} \int_{-H / 2}^{H / 2} u(y) d y
$$

and a slip boundary condition as, for example, that corresponding to the Navier slip model (Eq. 4),

$$
\mathbf{t} \cdot \mathbf{u}=-k \mathbf{t} \cdot \boldsymbol{\tau} \cdot \mathbf{n} \Rightarrow u_{s}(H / 2)=\left.k \mu \frac{\partial u}{\partial y}\right|_{y=H / 2} k \in[0 ;+\infty[
$$


resulting in:

$$
u(y)=\frac{U_{1}\left(y^{2}-k \mu H-\frac{H^{2}}{4}\right)}{\left(-\frac{H^{2}}{6}-k_{1} \mu H\right)} \quad k \in[0 ;+\infty[
$$

For assessment purposes, the results obtained with Eq. 12 and the corresponding numerical results are shown in Figure 3. As can be seen, the two set of results superimpose for the two examples considered.

The next case study consisted in testing the code with a more complex geometry (the final zone of an extrusion die) using again the Navier slip model and different friction coefficients. The corresponding velocity fields (global and at the die exit cross-section) are shown in Figure 4, together with the indication of the slip velocity occurring at a fix point located at the parallel zone of the die, i.e., at its more restrictive zone.

The simulations were performed for a constant flow rate that corresponds to an average extrusion velocity of $U=15.9 \mathrm{~mm} / \mathrm{s}$. As expected, the increase of the slip intensity, promotes an increase of the slip velocity through the entire channel wall, particularly at the most restrictive zones such as the parallel (final) zone of the die and the channel defined by two adjacent spider legs. From the outlet velocity distribution, it can also be seen that an increasing slip favors the uniformity of the flow distribution, which was expected since slip decreases the flow restriction promoted by the channel walls.

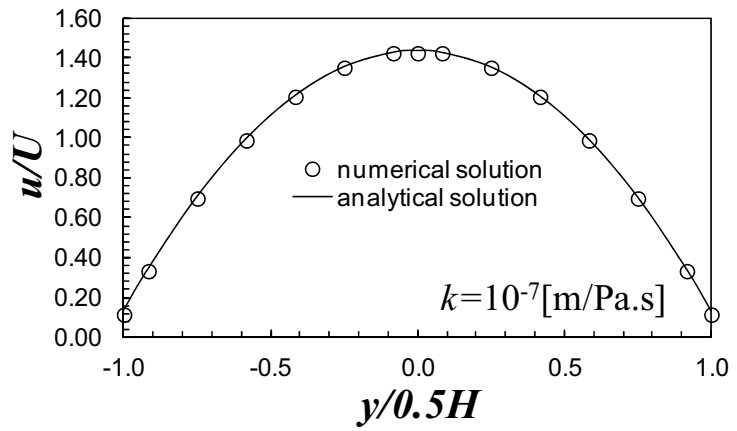

(a)

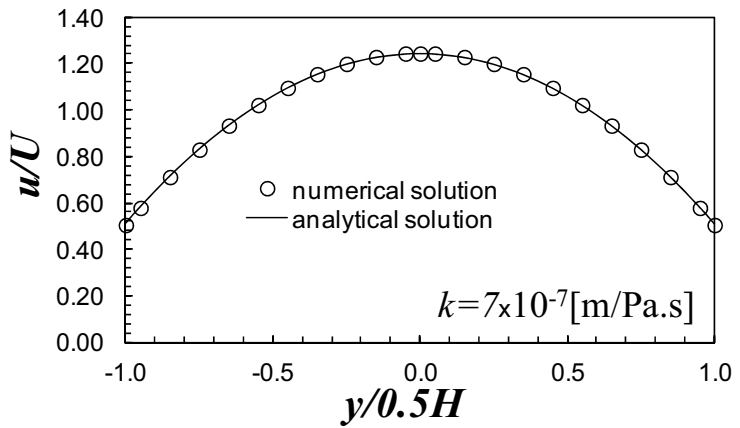

(b)

FIGURE 3. Comparison between the analytic and numerical solutions for the flow between parallel plates, using different slip coefficients: (a) $k=10^{-7}[\mathrm{~m} / \mathrm{Pa} . \mathrm{s}]$; (b) $k=7 \times 10^{-7}[\mathrm{~m} / \mathrm{Pa} . \mathrm{s}]$. 


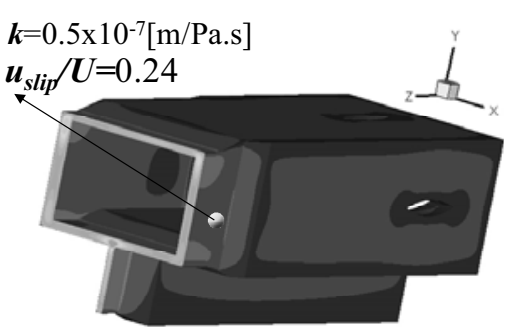

(a)

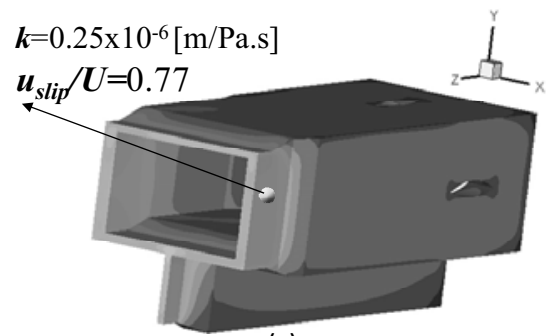

(c)

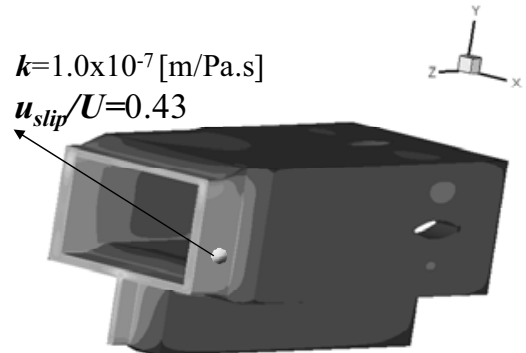

(b)

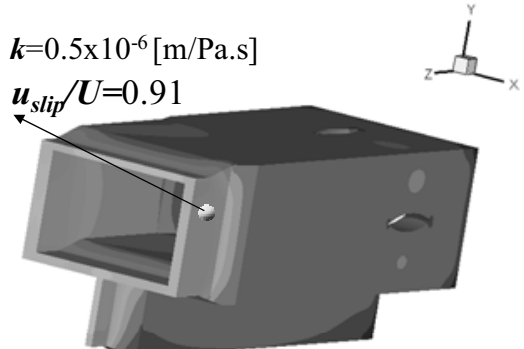

(d)

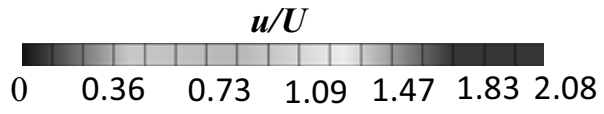

FIGURE 4. Velocity contours obtained for an extrusion die, using different slip coefficients $(k)$.

\section{OPTIMIZATION METHODOLOGY}

The optimization methodology used in this work was already described elsewhere and encompassed the implementation of two strategies [5]: i) the length strategy, where the controllable geometric parameters are the lengths of the die parallel zone, and, ii) the thickness strategy, where the thicknesses of the same zone are the controllable parameters.

The objective function used to evaluate the different trial geometries used by the algorithm [5], considers two criteria, one related to flow balance at the die exit (or velocity uniformity) and another concerning the $L / t$ of the parallel zone (related to the robustness of the die, i.e., its sensitivity to variations of the operating conditions and melt rheology), according to the equation bellow,

$$
F_{o b j}=\sum_{i=1}^{n_{Z}}\left\{\left\{\psi\left(1-\frac{V_{i}}{V_{o b j, i}}\right)^{2}+q(1-\psi)\left[1-\frac{(L / t)_{i}}{(L / t)_{\min }}\right]^{2}\right\} \frac{A_{o b j, i}}{A_{o b j}}\right\}
$$

where:

$n_{z}-$ total number of elemental (ES) and intersection (I) zones considered in the parallel zone;

$L / t$ - ratio between the length and thickness of each ES;

$q=0$ for all I zones and for ES zones with $L / t \geq 7$;

$q=1$ for ES zones with $L / t<7$; 
$V_{i}$ - actual average velocity of the melt flow in each zone;

$(L / t)_{\min }-$ minimum value recommended for the ratio $L / t$ (here considered to be 7 ); $\psi$ - relative weight;

$A_{o b j}, A_{o b j, i}$ - objective cross section areas of the global flow channel and of each zone, respectively;

$A_{i}$ - actual cross section area of each zone;

$\bar{V}$ - global flow average velocity;

$V_{o b j, i}$ - objective average velocity of the melt flow in each zone, given by the continuity equation:

$$
V_{o b j, i}=\bar{V} \frac{A_{o b j, i}}{A_{i}}
$$

Based on its definition, the quality of the trial die increases with the decrease of $F_{o b j}$, which reaches the value of 0 when the die is perfectly balanced with all ES lengths higher than the minimum recommended value.

\section{CASE STUDY}

The parallel zone cross section of the extrusion die to be optimized is shown in Figure 5. It is composed by several elemental sections (ES) of different thickness, ranging from 2 to $4 \mathrm{~mm}$, all other dimensions being presented in Table 1 . These values were imposed deliberately in order to promote different local flow restrictions.

As shown in Table 1, for the initial geometry the length/thickness $(L / t)$ of all ES was set equal to 15 . The operating conditions used were a flow rate of $20 \mathrm{~kg} / \mathrm{h}$ and a melt inlet temperature of $230^{\circ} \mathrm{C}$. The rheological behavior considered for the non-slip case optimization was that of a real polymer (a PP homopolymer); to have into account the slip at the die walls, the Navier slip model was considered.

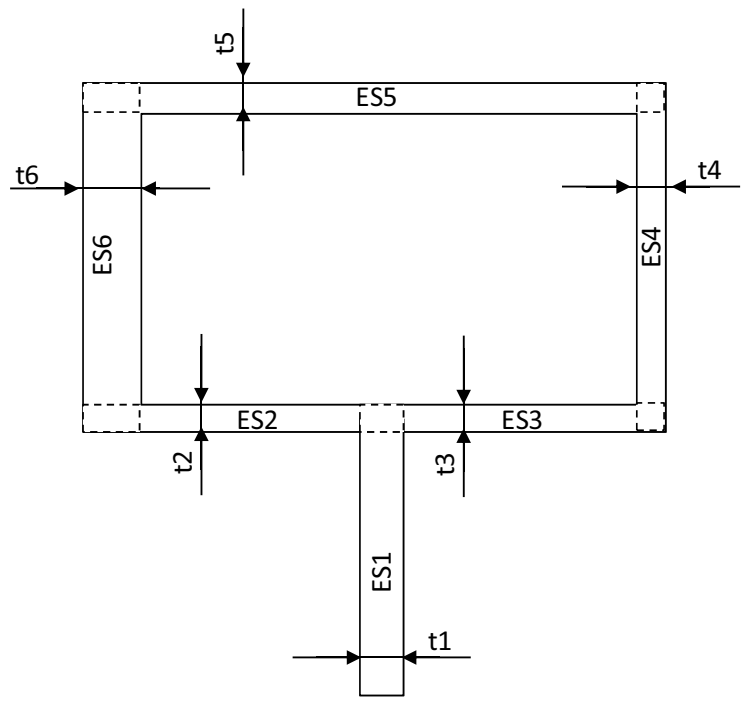

FIGURE 5. Cross-section of the parallel zone of the die used as a case study. 
TABLE 1. Initial flow channel dimensions.

\begin{tabular}{cccccc}
\hline ES & 1 & $2-3$ & 4 & 5 & 6 \\
\hline$t_{i}[\mathrm{~mm}]$ & 2.0 & 2.5 & 3.0 & 2.0 & 4.0 \\
$L_{i}[\mathrm{~mm}]$ & 30.0 & 37.5 & 45.0 & 30.0 & 60.0 \\
$L_{\mathrm{i}} / t_{i}$ & 15.0 & 15.0 & 15.0 & 15.0 & 15.0 \\
\hline
\end{tabular}

A total of four optimizations were performed, two for each optimization strategy (length and thickness), considering adherence and slippage of the polymer melt at the die walls.

\section{RESULTS AND DISCUSSION}

\section{Length Strategy}

After optimization, the lengths of the ES zones resulted in the values shown in Table 2. It is evident that the strategy adopted in the optimization resulted in drastic reductions of the initial lengths of the ES for the two optimized dies (designated as noslip and slip). In fact, five of these zones have now $L / t$ values lower than the minimum value recommended (here considered to be 7), being, however, these values slightly higher for the die optimized with the slip boundary condition.

TABLE 2. Dimensions of the optimized extrusion dies, using the length strategy, for the two cases considered (no-slip and slip at the die walls).

\begin{tabular}{c|cccccc}
\hline \multicolumn{2}{c}{ ES } & 1 & $2-3$ & 4 & 5 & 6 \\
\hline \multicolumn{2}{c}{$t_{i}[\mathrm{~mm}]$} & 2.0 & 2.5 & 3.0 & 2.0 & 4.0 \\
\hline$L_{i}[\mathrm{~mm}]$ & \multirow{2}{*}{ No-slip } & 7.5 & 11.5 & 17.5 & 7.0 & 60.0 \\
$L_{i} / t_{i}$ & \multirow{2}{*}{3.8} & 4.6 & 5.8 & 3.5 & 15.0 \\
\hline$L_{i}[\mathrm{~mm}]$ & \multirow{2}{*}{ Slip } & 8.5 & 12.5 & 19 & 8.5 & 60 \\
$L_{i} / t_{i}$ & & 4.3 & 5.0 & 6.3 & 4.3 & 15.0 \\
\hline
\end{tabular}

Concerning flow distribution (see Figure 6), it can be seen that slippage promotes a better flow distribution. The values of the objective function corresponding to the two optimized dies are 0.0322 and 0.0200 for the dies optimized with the non-slip and slip boundary condition, respectively. Having in mind the results shown, it can be concluded that when slippage occurs the objective function improves as a consequence of two factors: higher uniformity of the velocities at the die exit and higher $L / t$ values of its parallel zone. 


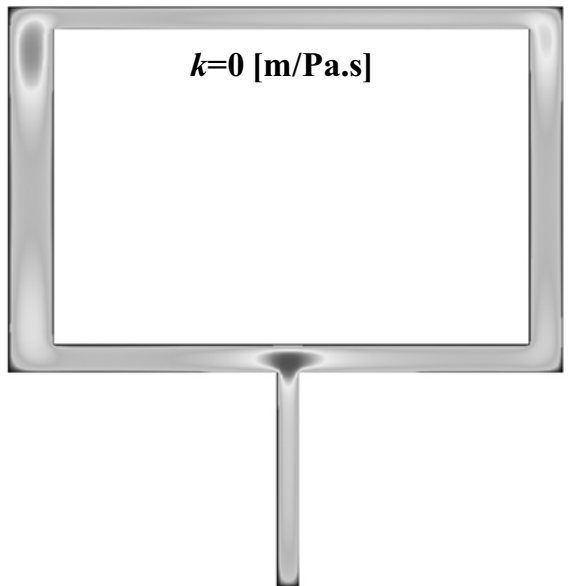

(a)

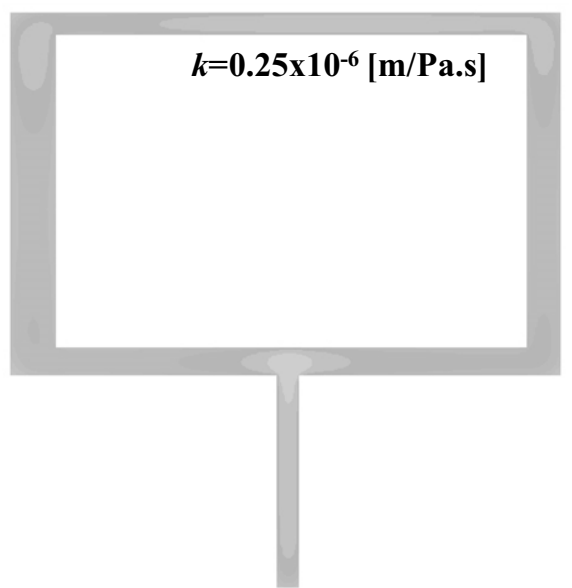

(b)

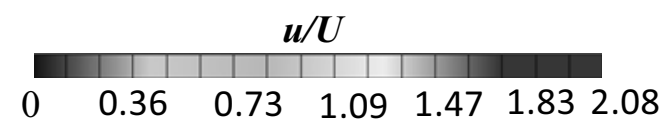

FIGURE 6. Velocity fields at the extrusion die outlet cross-section for the length strategy optimized dies, considering: (a) no-slip boundary condition; (b) slip boundary condition $k=0.25 \times 10^{-6}[\mathrm{~m} / \mathrm{Pa} . \mathrm{s}]$.

The occurrence of slip has other implications (advantages) such as the reduction of the total pressure drop and the limited heat viscous dissipation experienced by the melt (see Figure 7).

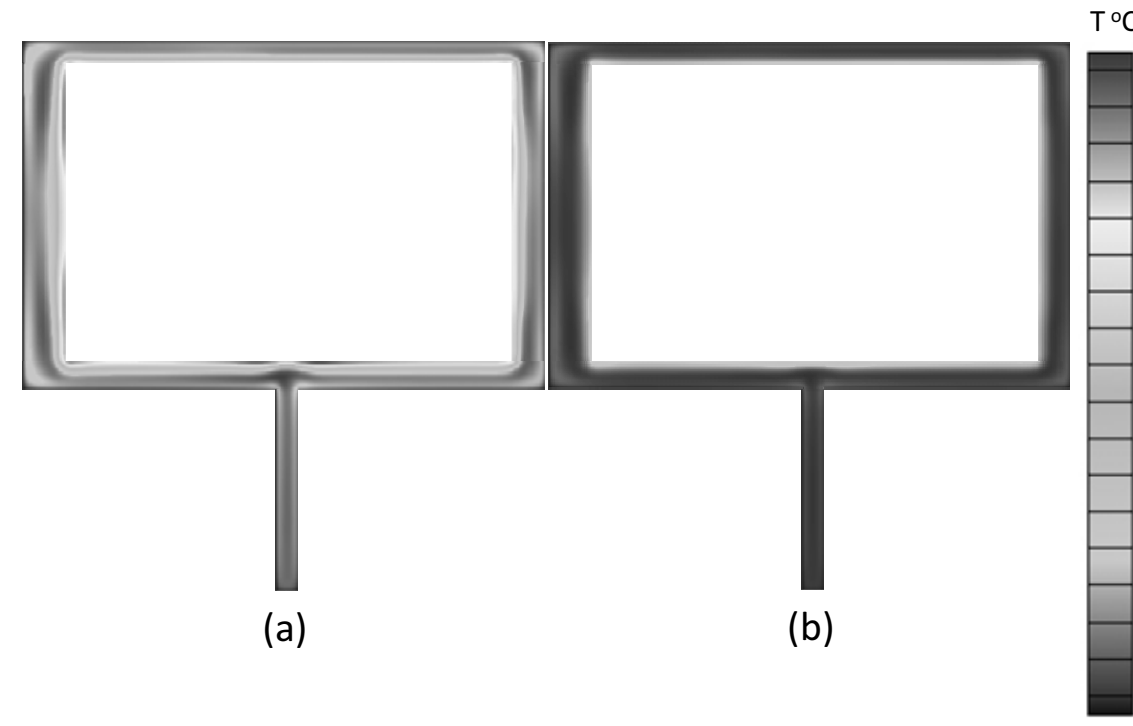

233.8

232.9

232.1

231.1

230.2

FIGURE 7. Temperature fields at the extrusion die outlet cross-section for the length strategy optimized dies, considering: (a) no-slip boundary condition; (b) slip boundary condition $k=0.25 \times 10^{-6}[\mathrm{~m} / \mathrm{Pa} . \mathrm{s}]$. 


\section{Thickness Strategy}

Again, and as expected, the flow of the optimized die with the slip boundary condition is more balanced than the one optimized assuming the no-slip (see Figure 8). In this case, the additional beneficial effect of slip consists in thicknesses of the final optimized die closer to those of the profile to be produced, as shown in Figure 9, which will diminish the differential pulling of the extrudate during production and its consequent lower propensity to distort in service, as discussed in a previous work [5].

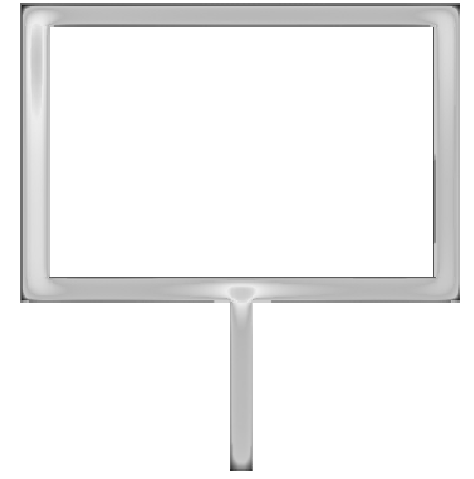

(a)

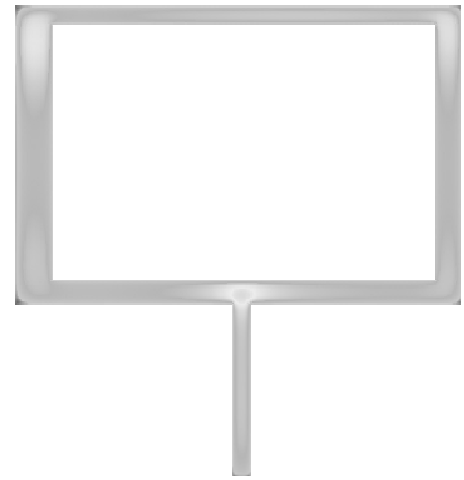

(b)

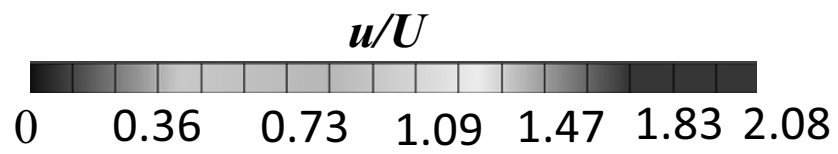

FIGURE 8. Velocity fields at the extrusion die outlet cross-section for the thickness strategy optimized dies, considering: (a) no-slip boundary condition; (b) slip boundary condition $k=0.25 \times 10^{-6}[\mathrm{~m} / \mathrm{Pa} . \mathrm{s}]$.

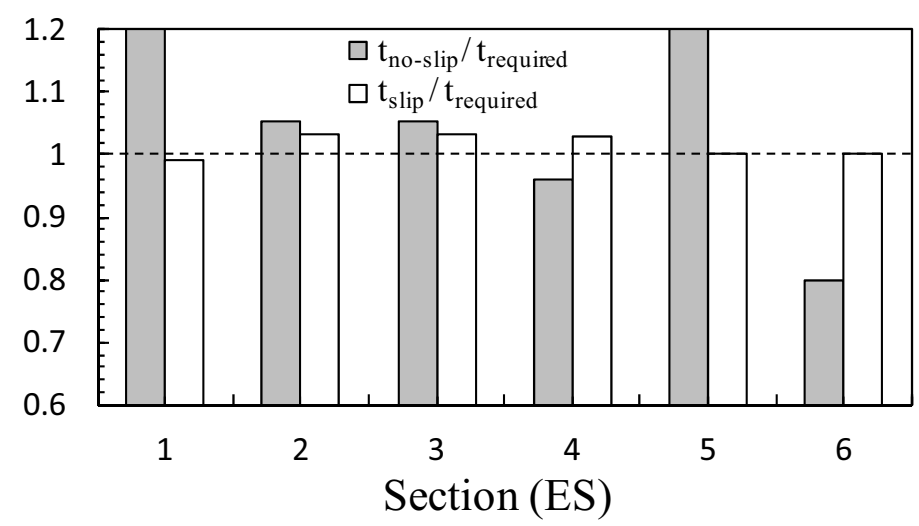

FIGURE 9. Ratio between optimized and required ES thickness $\left(t_{\text {required }}\right)$, for the dies optimized using the thickness strategy, for the two cases considered (no-slip and slip boundary condition). 


\section{CONCLUSION}

In this work the implementation of the slip boundary condition at the flow channel walls in a 3D finite volume numerical code previously developed was described and verified. The potential of the new numerical code was illustrated with a case study, where the observed variations of the slip velocity were in accordance with the expected. The flow balance optimization studies performed, allowed the conclusion that, in general terms, slippage improves the performance of extrusion dies, contributing for a more balanced flow distribution, independently of the optimization strategy used. When the length strategy is employed, higher lengths are obtained for the more restrictive zones (ES), thus the resulting die is expected to be less sensitive to variations of the operating conditions/polymer rheology. On the other hand, if the thickness strategy is used, the thicknesses obtained for the different ES of the die parallel zone are closer to those required for the extruded profile, thus diminishing the differential pulling of the extrudate during production and, consequently, the potential tendency of the profile to distort in service.

\section{ACKNOWLEDGMENTS}

The authors gratefully acknowledge funding from Fundação para a Ciência e Tecnologia, FCT (COMPETE Program) under the projects FCOMP-01-0124 FEDER-010190 (Ref. PTDC / EME - MFE/102729/2008) and FCOMP-01-0124FEDER-015126 (Ref ${ }^{\mathrm{a}}$. FCT PTDC/EME-MFE/113988/2009), and FEDER, via FCT, under the PEst-C/CTM/LA0025/2011 (Strategic Project - LA 25 - 2011-2012).

\section{REFERENCES}

1. J. M. Nóbrega, O. S. Carneiro, F. T. Pinho and P. J. Oliveira, Int. Polym. Process. 19, 225-235 (2004).

2. M. Mooney, J. Rheol. 2, 211-222 (1931).

3. S. G. Hatzikiriakos and J. M. Dealy, J. Rheol. 36, 703-741 (1992).

4. S. G. Hatzikiriakos and J. M. Dealy, J. Rheol. 35, 497-523 (1991).

5. J. M. Nóbrega, O. S. Carneiro, P. J. Oliveira and F. T. Pinho, Int. Polym. Process. 18, 298-306 (2003).

6. L. L. Ferrás, J. M. Nóbrega and F. T. Pinho, J. Non-Newtonian Fluid Mech. 175-176, 76-88 (2012).

7. L. L. Ferrás, J. M. Nóbrega and F. T. Pinho, J. Non-Newtonian Fluid Mech. 171-172, 97-105 (2012).

8. L. L. Ferrás, J. M. Nóbrega and F. T. Pinho, Int. J. Numer. Meth. Fluids doi:10.1002/fld.3765.

9. J. Heywood, R. Rannacher and S. Turek, Int. J. Numer. Meth. Fluids 22, 325-352 (1996).

10. E. Lauga, M. P. Brenner and H. A. Stone, "Chapter 15: Microfluidics: The No-Slip Boundary Condition" in Handbook of Experimental Fluid Dynamics, edited by C. Tropea, A. L. Yarin, J. F. Foss, New York: Springer, 2005, pp. 1219-1235.

11. S. Granick, Y. X. Zhu and H. Lee, Nature Mat. 2, 221-227 (2003).

12. P. Tabeling, C. R. Physique 5, 531-537 (2004).

13. H. Lamb, Hydrodynamics, New York: Dover, 1932.

14. G. K. Batchelor, An Introduction to Fluid Dynamics, Cambridge: Cambridge University Press, 1967. 
15. S. Goldstein, Modern Developments in Fluid Dynamics: An Account of Theory and Experiment Relating to Boundary Layers, Turbulent Motion and Wakes, Oxford: Oxford University Press, 1938.

16. M. M. Denn, Annu. Rev. Fluid Mech. 33, 265-287 (2001).

17. S. G. Hatzikiriakos, Int. Polym. Process. 8, 135-142 (1993).

18. H. Potente, H. Ridder and R. V. Cunha, Macromol. Mat. Eng. 287, 836-842 (2002).

19. E. Mitsoulis, I. B. Kazatchkov and S. G. Hatzikiriakos, Rheol. Acta 44, 418-426 (2005).

20. W. J. Silliman and L. E. Scriven, J. Comp. Physics 34, 287-313 (1980).

21. A. Liakos, Computer and Mathematics with Applications 48, 1153-1166 (2004).

22. A. Liakos, Computers and Mathematics with Aplications 49, 281-294 (2005).

23. ANSYS POLYFLOW CFD (http://www.ansys.com).

24. M. Peric, "A Finite Volume Method for the Prediction of Three-Dimesional Fluid Flow in Complex Ducts", Ph.D. Thesis, University of London, 1985. 